\title{
Fortuitous Discovery of a Left Atrial Myxoma Following Acute Myocardial Infarction
}

\author{
Major N A Hoad, \\ MA, MB, BChir, MRCP RAMC \\ Senior Specialist in Medicine* \\ WO I J S Foulds, \\ RAMC \\ * Department of Physiological Measurement, Cambridge Military Hospital, Aldershot, Hants
}

SUMMARY: Routine echocardiography following acute myocardial infarction revealed a large totally asymptomatic left is atrial myxoma. One year after excision of the myxoma and coronary artery by-pass grafting, the patient remains well.

\section{Introduction}

Although the commonest cardiac tumour, atrial myxoma is still a rarity ${ }^{1}$. This report is believed to be the first case of an atrial myxoma being diagnosed quite unexpectedly at routine echocardiography following acute myocardial infarction.

\section{Case Report}

A 68 year old caucasian female was admitted to the coronary care unit with a one hour history of severe retrosternal chest pain, associated with sweating, nausea and dyspnoea. There was no past history of ischaemic heart disease nor of hypertension. She smoked 20 cigarettes daily and was on no regular medication.

On examination her pain had been greatly relieved by an opiate. She was not shocked, had a pulse rate of $104 / \mathrm{m}, \mathrm{BP} 140 / 100 \mathrm{mmHg}$ and there was no elevation of the jugular venous pressure. There was a third heart sound, a short systolic murmur at the left sternal edge without radiation, and bilateral basal crackles. ECG revealed sinus rhythm and ST elevation in the inferior leads. Chest X-ray revealed a normal sized heart with upper lobe diversion of blood but no pulmonary oedema.

A diagnosis of inferior myocardial infarction was made together with early left ventricular failure, and treatment with a loop diuretic and further analgesia was commenced. Subsequent ECG's evolved to show a full thickness inferior infarction and the serum creatine kinase peaked at five times the upper limit of normal.

The sinus tachycardia settled and there were no arrhythmic complications. The basal crackles disappeared within forty-eight hours, although the third sound and murmur persisted. She mobilised steadily with no recurrence of chest pain and appeared to be making an uneventful recovery.

One week post infarction, routine two dimensional echo-cardiography was performed. This revealed a large mass in the left atrium which prolapsed through the mitral valve in diastole (Fig 1). These are the typical

*Now at Queen Elizabeth Military Hospital, Woolwich. features of a myxoma. On direct questioning however she denied any relevant symptoms of constitutional upset, embolic cvents or exercise induced dyspnoea. She was not anaemic and her immunoglobulins were not elevated. Her ESR was very high at $106 \mathrm{~mm}$ in the first hour.

She was referred for further assessment. Two months later, at cardiac catheterisation, the echocardiograph findings were confirmed. Triple vessel coronary artere? 윽 disease was also present with $70 \%$ stenosis of the left anterior descending artery, $80 \%$ stenosis of the्ष्त circumflex artery, and $70 \%$ stenosis of the right coronaro artery. ESR at that time was $80 \mathrm{~mm}$ in the first hour. Te days later, at surgery, a large left atrial myxom; attached to the inter-atrial septum by a small stalk, wa्र $\overrightarrow{0}$ cxcised; this was followed by triple vessel coronafo artery by-pass grafting.

She made a good post-operative recovery, arf remains well one year later with no angina, no abnormal clinical findings and a normal echocardiogram and ESR.

\section{Comment}

At this hospital, two dimensional echocardiography is performed routinely on all patients following myocardial infarction. This is done both to assess left ventricular function and to exclude intra-cavity clot and pericardial effusion. The findings in this patient were totally unexpected, and without this investigation the myxoma would have been missed. The ESR is not usually measured, and the persistent third heart sound, which may in retrospect have been a so-called "tumour plop", was attributed to the recent infarction. One study however failed to detact any "tumour plops" in a series of ten myxomas ${ }^{2}$.

What were the advantages to the patient of diagnosing an asymptomatic myxoma in this way? These tumours are thought to be slow growing ${ }^{3}$, and must initially be asymptomatic. Many are diagnosed at a later stage with the typical history of obscure cardiac symptoms, chronic constitutional disturbance or embolic events ${ }^{4}$. Acute catastrophes however also occur. With larger tumours 
these may be sudden obstruction of the atrio-ventricular valves ${ }^{5}$ or embolism. This latter complication is also seen with smaller tumours ${ }^{2}$. It is possible that this patient had a coronary artery embolus, but the presence of diffuse triple vessel disease makes this unlikely. Early surgery for atrial myxoma is now recommended after diagnosis'; indeed delay between diagnosis and surgery has been fatal $^{6}$.

In this case, surgery was delayed for three months. This was to diminish the known increased risks of surgery following a myocardial infarction. Her prognosis should be good. Long term follow up studies for nearly twenty years suggest that the risk of recurrence is low ${ }^{1}$, and that regular two-dimensional echocardiography is a simple and effective way of screcning ${ }^{6}$.

No other example of left atrial myxoma being diagnosed in this way following an acute myocardial infarction has been found. As coronary artery disease is common, the two conditions have been reported to coexist $^{8}$. However in such patients the myxoma was the primary diagnosis and the coronary artery disease was discovered during pre-operative assessment. Echocardiography is a valuable investigation following a myocardial infarction, but to diagnose a myxoma was an unexpected bonus.

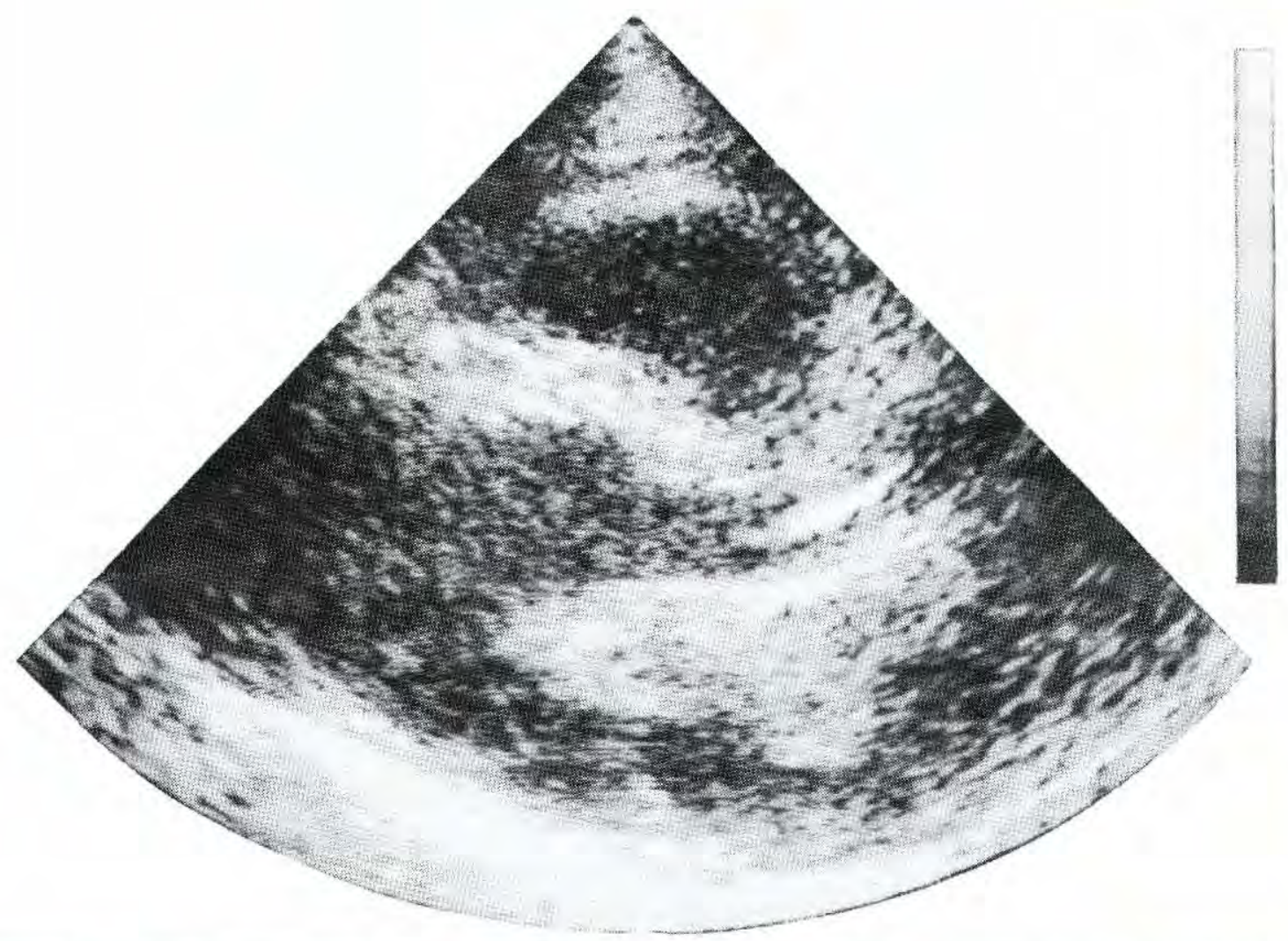

\section{4}
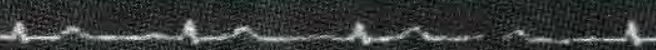

Fig 1. (a) Long axis echocardiograph showing myxoma in left antrium. 

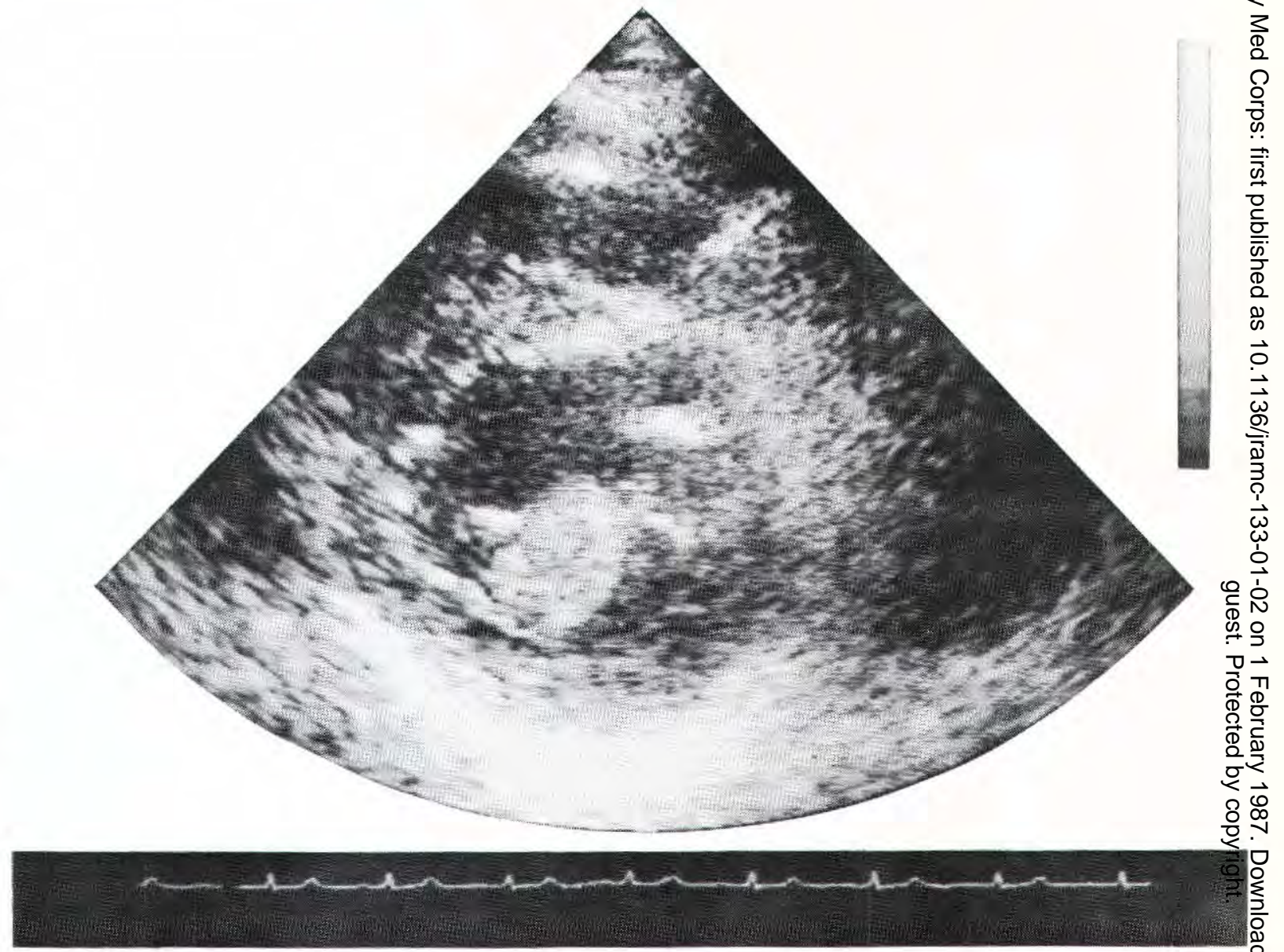

Fig 1. (b) Short axis echocardiograph showing myxoma prolapsing through mitral valve.

\section{REFERENCES}

1. Rosman H S et al. Two decades of atrial tumour. Eur Heart Journal 1982; 3: 100-106.

2. Attar S et al. Cardiac Myxoma. Ann Thorac Surg 1980: 29: $397-405$.

3. Oldershaw P J, St John Sutton M and Gibson R V. Long asymptomatic period of atrial myxomas. Thorax 1980; 35: 70-71.

4. Goodwin J F. Diagnosis of left atrial myxoma. Lancet 1963; 1: $464-467$.
5. O'NFil. M B, Grehl T M and Hurley E J. Cardiac $\frac{3}{3}$ myxomas, a clinical diagnostic challenge. Am J Surg $1979 ; 138: 68-76$.

6. L.rvi U et al. Cardiac myxomas: results of 14 years experience. J Thorac Cardiovasc Surgery 1984; 32:윽 143-147.

7. SEMB B K H. Surgical considerations in the treatment of cardiac myxoma. I Thorac Cardiovasc Surgery 1984; 87: N
251-259.

8. RICE P L and PIFARRE R. Left atrial myxoma and N coronary artery disease. Arch Surg 1981; 116: 353-355. 